\title{
The onset of the GeV afterglow of GRB 090510
}

\author{
G. Ghirlanda ${ }^{1}$, G. Ghisellini ${ }^{1}$, and L. Nava ${ }^{2}$ \\ 1 INAF-Osservatorio Astronomico di Brera, via E. Bianchi 46, 23807 Merate, Italy \\ e-mail: giancarlo.ghirlanda@brera.inaf.it \\ 2 SISSA-ISAS, via Beirut 2-4, 34151 Trieste, Italy
}

Received 29 December 2009 / Accepted 21 January 2010

\section{ABSTRACT}

\begin{abstract}
We study the emission of the short/hard Gamma Ray Burst 090510 at energies $>0.1 \mathrm{GeV}$ as observed by the Large Area Telescope (LAT) onboard the Fermi satellite. The GeV flux rises in time as $t^{2}$ up till $0.2 \mathrm{~s}$ after the peak of the MeV pulse detected by the Fermi Gamma Burst Monitor (GBM) after which it decays as $t^{-1.5}$ up to $200 \mathrm{~s}$. Its energy spectrum is consistent with $F(v) \propto v^{-1}$. The time behavior and the spectrum of the high energy LAT flux are strong evidences of an afterglow origin. We then interpret it as synchrotron radiation produced by the forward shock of a fireball with a bulk Lorentz factor $\Gamma \sim 2000$. The afterglow peak time is independent of energy in the $0.1-30 \mathrm{GeV}$ range and coincides with the arrival time of the highest energy photon $(\sim 30 \mathrm{GeV})$. Since the flux detected by the GBM and the LAT have different origins, the delay between these two components is not entirely due to possible violation of the Lorentz invariance. The LAT component alone allows us to set a reliable lower limit on the quantum-gravity mass of 4.7 times the Planck mass.
\end{abstract}

Key words. gamma-ray burst: general - radiation mechanisms: non-thermal - X-rays: general

\section{Introduction}

The EGRET instrument onboard the Compton Gamma Ray Observatory (CGRO) detected in few gamma ray bursts an emission above $100 \mathrm{MeV}$ (Fishman \& Meegan 1995; Kaneko et al. 2008). Since 2008 the Large Area Telescope (LAT) on board the Fermi telescope with its much better sensitivity (and reduced dead time) has detected 12 GRBs in the $100 \mathrm{MeV}$ to few $\mathrm{GeV}$ energy range. These bursts can shed light for the first time on the origin of the $\mathrm{GeV}$ emission and will help to answer some of the open questions of which, the most compelling one is probably that about its origin, i.e. does the $\mathrm{GeV}$ emission belong to the prompt phase, or is it afterglow emission produced by the fireball colliding with the circum-burst medium? Or does it have yet another origin?

GRB 090510 is a short/hard burst at redshift $z=0.903 \pm$ 0.003 (Rau et al. 2009) detected by Fermi (Guiriec et al. 2009), AGILE (Longo et al. 2009), Swift (Hoversten et al. 2009), Konus-Wind (Golenetskii et al. 2009) and Suzaku (Ohmori et al. 2009).

The Fermi-Gamma Burst Monitor (GBM) triggered on a precursor while the main emission episode in the $8 \mathrm{keV}-40 \mathrm{MeV}$ energy range started $\sim 0.5 \mathrm{~s}$ after trigger and lasted up to $\sim 1 \mathrm{~s}$. The emission observed by the Fermi-Large Area Telescope (LAT) started $0.65 \mathrm{~s}$ after the trigger and lasted $200 \mathrm{~s}$. The joint GBM-LAT spectral analysis showed the presence of two components. Fermi-LAT detected a $31 \pm 3 \mathrm{GeV}$ photon delayed by $0.829 \mathrm{~s}$ with respect to the trigger (Abdo et al. 2009a - A09 hereafter).

The precursor was not seen by AGILE, which triggered on the main emission episode. The flux detected by the Mini Calorimeter (MCAL, $0.3-10 \mathrm{MeV}$ ) lasted $0.2 \mathrm{~s}$. As it ended, the Gamma Ray Imaging Detector (GRID, 0.03-30 GeV) started to detect a high energy component lasting for $10 \mathrm{~s}$ and decaying as $t^{-1.3}$ (Giuliani et al. 2009).

The distinguishing property of GRB 090510 arising from the Fermi and AGILE data is that the $\sim \mathrm{MeV}$ emission component, commonly detected in GRBs, was followed by a much longer lasting high energy emission detected above $100 \mathrm{MeV}$. Both the AGILE and Fermi spectra suggested that this component is not the extrapolation of the soft $\sim \mathrm{MeV}$ spectrum to the $\mathrm{GeV}$ range. A09 interpreted the $\sim \mathrm{MeV}$ flux as synchrotron radiation and the LAT flux as its synchrotron self-Compton emission. The detection by Fermi of a $30 \mathrm{GeV}$ photon allowed us to set a lower limit on the bulk Lorentz factor of the fireball $\Gamma>1000$, based on the compactness argument (A09). The $30 \mathrm{GeV}$ photon arrived $0.829 \mathrm{~s}$ after the trigger (set by the precursor) and $0.3 \mathrm{~s}$ after the beginning of the GBM main pulse. A09 interpreted the flux detected by the LAT as due to the self-Compton scatterings of the lower frequencies synchrotron photons detected by the GBM. In this framework, the GBM and LAT detected photons are produced in the same region. Their emission time can then be the same. If true, this allows us to interpret the delayed arrival time of the $31 \mathrm{GeV}$ photon as due to Lorentz invariance violation. This, in fact, introduces an energy dependent photon speed. A09 themselves, however, consider also other hypotheses (i.e. different delay times) and derive several lower limits on the quantum-gravity mass scale.

Recent works on the high energy emission of LAT-detected GRBs include Kumar \& Barniol Duran (2009a,b), discussing GRB 080916C (Abdo et al. 2009b). For this burst they also proposed that the LAT-detected flux can be synchrotron produced in the external shock (as suggested by Gao et al. 2009; Corsi et al. 2009a,b; and De Pasquale et al. 2009 for GRB 090510; but see also Fan et al. 2008; Zou et al. 2009; Zhang \& Peer 2009, for an inverse Compton origin). Hadronic models have been proposed 


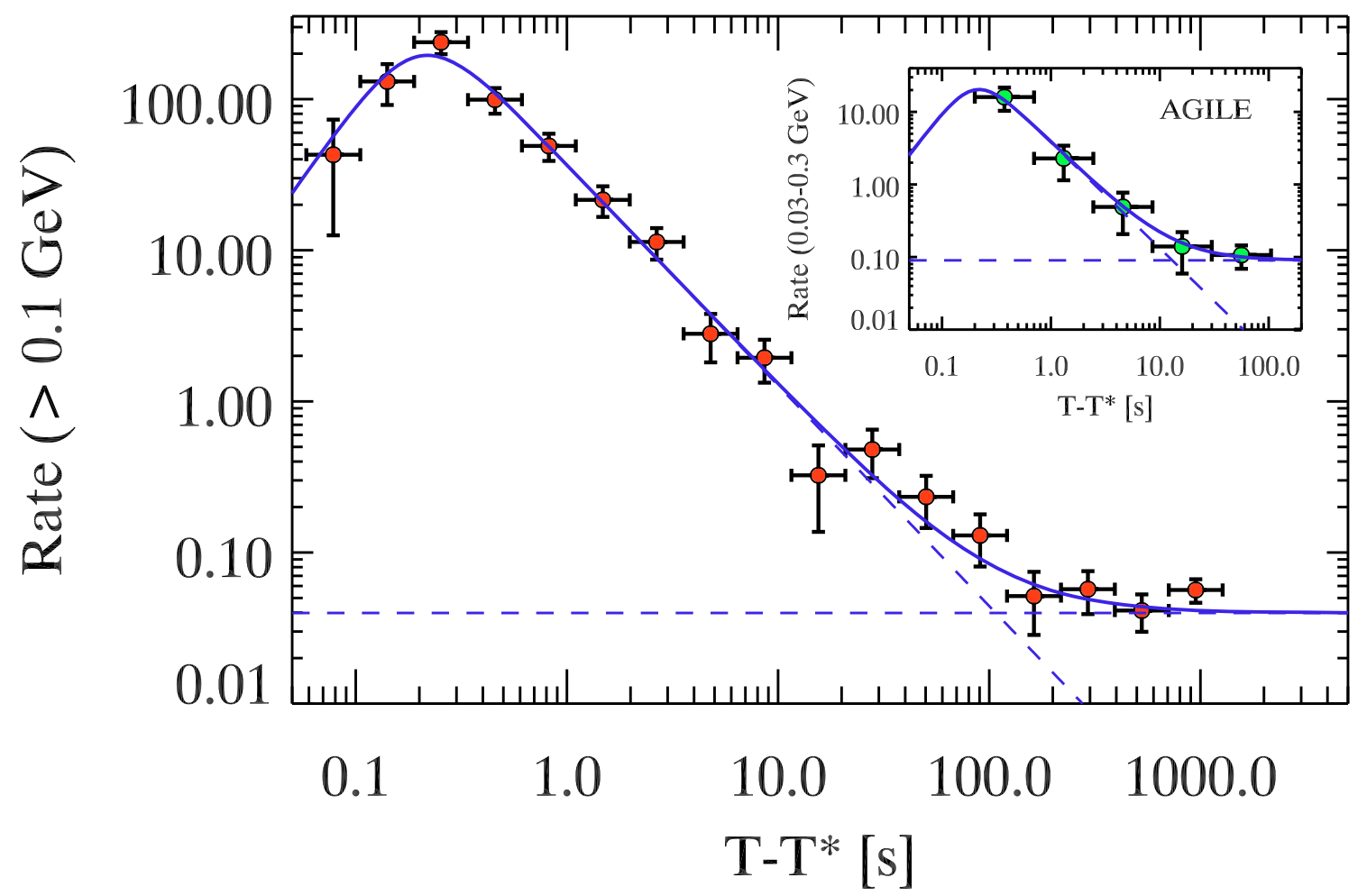

Fig. 1. Fermi-LAT light curve of the emission of GRB 090510 above $100 \mathrm{MeV}$. The times were scaled to the time $T^{*}=0.6 \mathrm{~s}$ after the GBM trigger. This is the time corresponding to the main pulse of emission detected by the GBM in the $8 \mathrm{keV}-10 \mathrm{MeV}$ energy range (see A09). The solid line is the best fit to the data points obtained with a smoothly broken power-law plus a constant (dashed lines). The inset shows the AGILE light curve (photons energies between 30 and $300 \mathrm{MeV}$ ). The curve is the best fit of the Fermi data scaled to the AGILE data points.

to explain the emission properties of GRB 080916C (Razzaque et al. 2009; Asano et al. 2009) and a possible interpretation in the context of the cannonball model (Dado \& Dar 2009) has also been considered. A more extensive study of the light curve decay of the bursts detected by Fermi-LAT and on its possible interpretation in the context of the external shock produced by a radiative decelerating fireball is presented in Ghisellini et al. (2009).

If $\Gamma>1000$, the fireball should start to decelerate and produce a luminous afterglow rather early (e.g. Piran 2005), even at the sub-second timescale. We present by analyzing the FermiLAT light-curve and spectra of GRB 090510 we present strong evidences that the flux detected by the LAT is afterglow emission of the forward external shock.

In this framework we derive the initial $\Gamma$ of the fireball. According to our interpretation, the GBM and the LAT detected fluxes are produced in different regions and at different emission times. Therefore, the LAT emission by itself is the best tool to constrain the arrival time delay of photons of different (high) energies and derive a more reliable lower limit on the Lorentz invariance violation.

\section{Fermi-LAT data analysis}

We analyzed the Fermi-LAT data of GRB 090510 with the Fermi ScienceTools (v9r15p2) released on Aug. 8th 2008. Photons were selected (with the gtselect tool) around $\mathrm{RA}=333.552^{\circ}$ and Dec $=-26.598^{\circ}$. Different energy bins were considered for the analysis of the LAT light curve, but only photons with energy $>100 \mathrm{MeV}$ were extracted. Light curves and spectra were created with the gtbin tool. The spectral response files were created with the gtrspgen. The spectra were analyzed with $\mathrm{Xspec}(\mathrm{v} .12)$.

\section{Results}

Figure 1 shows the light curve considering all LAT photons with energies $>100 \mathrm{MeV}$. The times are scaled to $T^{*}=0.6 \mathrm{~s}$, which corresponds to the time of the first main pulse observed by the GBM. We fit the light curve with the sum of two components, i.e. a smoothly broken power law and a constant to account for the flattening of the flux visible after $200 \mathrm{~s}$, corresponding to the the time when the source flux becomes consistent with the background level (as also shown in De Pasquale et al. 2009):

$R(t)=\frac{A\left(t / t_{\mathrm{b}}\right)^{\alpha}}{1+\left(t / t_{\mathrm{b}}\right)^{\alpha+\beta}}+B$.

When $\alpha>0$ and $\beta>0$, Eq. (1) has a peak at $t_{\text {peak }}=$ $t_{\mathrm{b}}(\alpha / \beta)^{1 /(\alpha+\beta)}$. The standard afterglow theory (Sari \& Piran 1999) requires $\alpha=2$. Fixing $\alpha=2$, the best-fit parameters $\left(\chi^{2}=14.6 / 14\right)$ are $A=385_{-40}^{+45}$ counts $/ \mathrm{s}, t_{\mathrm{pk}}=0.217 \pm 0.015 \mathrm{~s}$, $\beta=1.46_{-0.03}^{+0.06}$ and $B=4 \times 10^{-2}$ counts/s. The best fit is shown by the solid line in Fig. 1. The AGILE light curve (adapted from Giuliani et al. 2009) of the photons detected by the GRID between $30 \mathrm{MeV}$ and $300 \mathrm{MeV}$ is also shown in Fig. 1 (inset). The Fermi and AGILE light curves are consistent with the same decay law, i.e. $t^{-1.5}$, although AGILE missed the rising phase of the $\mathrm{GeV}$ emission.

The emission above $100 \mathrm{MeV}$ peaks at $T-T^{*}=0.22 \mathrm{~s}$ (i.e. $0.82 \mathrm{~s}$ after the GBM trigger). The time of the peak coincides with the arrival time of the highest energy photon of $30 \mathrm{GeV}$. Figure 1 shows that the LAT flux lasts for about $200 \mathrm{~s}$ (and it sets to the background level afterwards). Instead, the emission detected by the GBM in the $8 \mathrm{keV}-10 \mathrm{MeV}$ energy range ceases after $\sim 1$ s (A09).

Figure 2 shows the LAT light curve in the first $10 \mathrm{~s}$ separated into two energy bands, i.e. $0.1-1 \mathrm{GeV}$ and $>1 \mathrm{GeV}$ (top 
G. Ghirlanda et al.: The onset of the GeV afterglow of GRB 090510

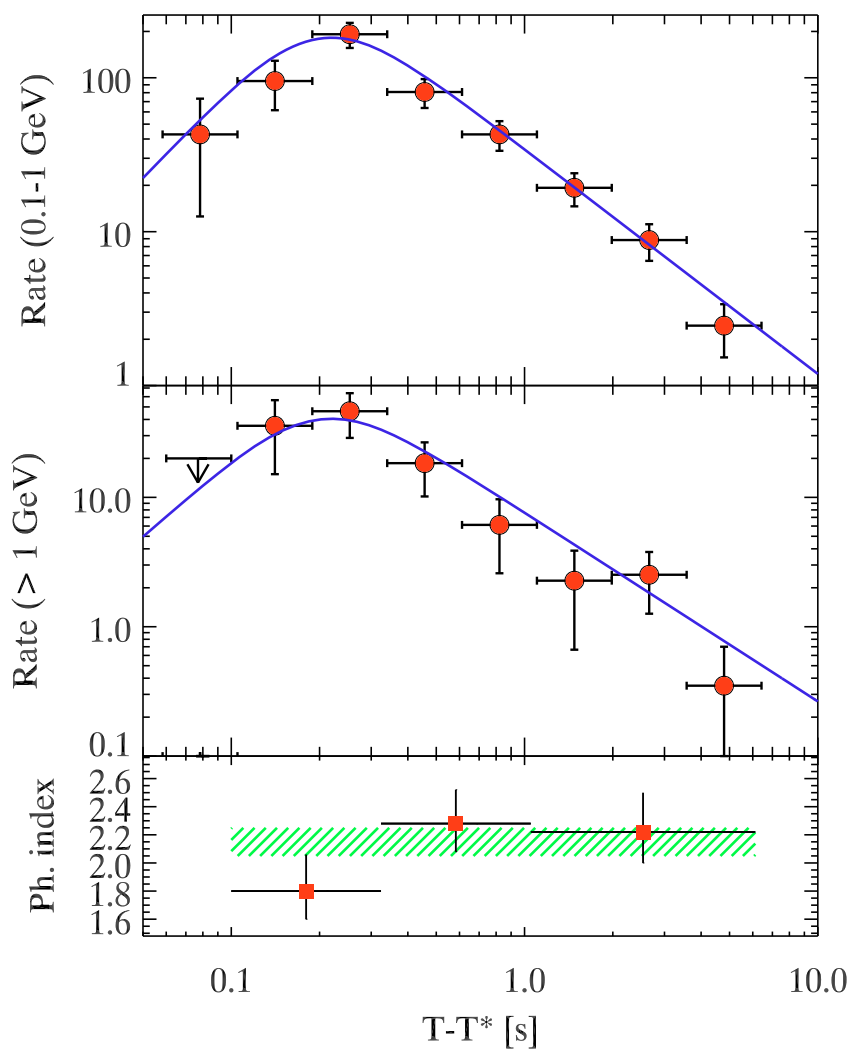

Fig. 2. Fermi-LAT light curve of GRB 090510 between 0.1 and $1 \mathrm{GeV}$ and above $1 \mathrm{GeV}$ (top and middle panels, respectively) in the first $10 \mathrm{~s}$. The times are scaled to $T^{*}=0.6 \mathrm{~s}$ (see text). The solid line is the fit of the light curve $>0.1 \mathrm{GeV}$ (Fig. 1). The bottom panel shows the photon spectral index ( $1 \sigma$ errors are shown) of the LAT spectra for the timeintegrated spectrum (hatched region) and for three time resolved spectra (squares).

and middle panels, respectively). The curves correspond to the same best fit obtained from the $>0.1 \mathrm{GeV}$ light curve (Fig. 1), only re-normalized to the data points. We further separated the light curve into four broad energy channels: $0.1-0.2 \mathrm{GeV}$, $0.2-0.4 \mathrm{GeV}, 0.4-0.8 \mathrm{GeV}$ and $>0.8 \mathrm{GeV}$ (Fig. 3). Figures 2 and 3 show that the time of the peak is the same in different energy ranges.

We also analyzed the spectra of the early $\mathrm{GeV}$ emission component. We considered the spectrum integrated in time between $T-T^{*}=0.1$ and $7 \mathrm{~s}$, and we also extracted three time resolved spectra distributed in this time interval. The photon spectral index of the fit with a single power law of the average spectrum (hatched region) and of the time resolved spectra (filled squares) are shown in the bottom panel of Fig. 2. The spectrum before the peak is hard with a photon index $1.87 \pm 0.2$ and then softens to $2.2 \pm 0.2$. The three time resolved spectra are consistent with the time-integrated spectrum.

\section{Estimate of the initial bulk Lorentz factor}

The derived peak time of the LAT received flux translates into an estimate of the bulk Lorentz factor $\Gamma_{0}$ at the start of the afterglow. The peak time of the afterglow bolometric luminosity occurs at a time of the order of the deceleration time. If the circumburst

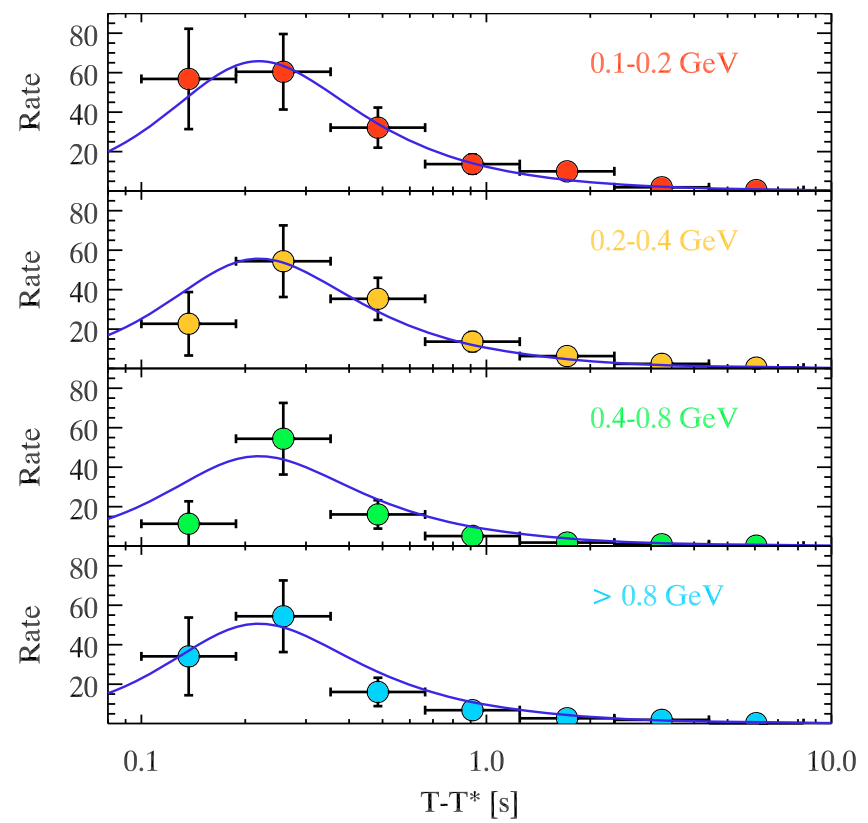

Fig. 3. Fermi-LAT light curve of GRB 090510 in four energy channels (from top to bottom): $0.1-0.2 \mathrm{GeV}, 0.2-0.4 \mathrm{GeV}, 0.4-0.8 \mathrm{GeV}$, $>0.8 \mathrm{GeV}$. The curves are the best fit obtained from the LAT light curve ( $>0.1 \mathrm{GeV}-$ Fig. 1) rescaled to the single channel light curves.

number density $n$ is homogeneous we have (e.g. Sari \& Piran 1999)

$\frac{t_{\text {peak }}}{1+z} \sim t_{\mathrm{dec}} \sim\left(\frac{3 E_{\mathrm{k}, \text { iso }}}{32 \pi n m_{\mathrm{p}} c^{5} \Gamma_{0}^{8}}\right)^{1 / 3}$,

where $E_{\mathrm{k} \text {,iso }}$ is the isotropic kinetic energy of the fireball, estimated through the emitted energy of the prompt emission assuming an efficiency $\eta\left(E_{\mathrm{k}, \text { iso }}=E_{\gamma \text {,iso }} / \eta\right)$. We use Eq. (2) to estimate $\Gamma_{0}$. Setting $E_{\gamma \text {,iso }}=3.5 \times 10^{52} \mathrm{erg}$ (A09, excluding the LAT component), $\eta=0.2$ and $t_{\text {peak }}=0.2 \mathrm{~s}$ we derive $\Gamma_{0}=1.96 \times 10^{3} n^{-1 / 8}$. This value is not much higher than the lower limits derived by A09 through the compactness argument and assuming that the LAT component belongs to the prompt emission. It is also rather insensitive to the (unknown) particle density $n$. The distance from the central engine corresponding to the peak time is $R_{\text {peak }} \sim 2 c t_{\text {peak }} \Gamma_{0}^{2} /(1+z)=2.4 \times 10^{16} n^{-1 / 4} \mathrm{~cm}$.

\subsection{A synchrotron origin of the LAT emission}

Following standard arguments, the minimum electron energy of the injected electrons in the forward shock is $\gamma_{\mathrm{m}} \sim \epsilon_{\mathrm{e}} \Gamma m_{\mathrm{p}} / m_{\mathrm{e}}$, while the magnetic field value is $B \sim \Gamma\left(8 \pi \epsilon_{\mathrm{B}} n m_{\mathrm{p}} c^{2}\right)^{1 / 2}$. Electrons with $\gamma_{\mathrm{m}}$ emit an observed synchrotron frequency $v_{\mathrm{m}} \sim$ $2 \Gamma(4 / 3) e B /\left(2 \pi m_{\mathrm{e}} c\right) \gamma_{\mathrm{m}}^{2} /(1+z) \sim 10.6 \Gamma_{3}^{4}\left(n \epsilon_{\mathrm{B}}\right)^{1 / 2} \epsilon_{\mathrm{e}}^{2} /(1+z) \mathrm{MeV}$. This frequency is below the LAT energy range, but the injection of a power law distribution of electrons extending to $\gamma_{\max } \sim$ $\left(10^{2}-10^{3}\right) \gamma_{\mathrm{m}}$ ensures that the LAT flux can indeed have a synchrotron origin. The synchrotron self-Compton (SSC) spectrum extends to much higher frequencies (e.g. Fan et al. 2008; Corsi et al. 2009a,b), but becomes important only above $v_{\mathrm{m}, \mathrm{C}} \sim \gamma_{\mathrm{m}}^{2} v_{\mathrm{m}}$, i.e. above the $\mathrm{TeV}$ energy range. Note the strong dependence of $v_{\mathrm{m}}$ and $v_{\mathrm{m}, \mathrm{C}}$ on the bulk Lorentz factor: a synchrotron origin of a $\sim \mathrm{GeV}$ afterglow is reasonable only for rather large $\Gamma_{0}$, while the SSC flux becomes more important for smaller $\Gamma_{0}$.

This has a simple and important consequence. Bursts with $\Gamma_{0} \sim 100$ or smaller can produce high energy afterglow 
radiation through the $\mathrm{SSC}$ mechanism, but the onset time of their afterglows will be large, in turn implying for the same emitted energy a lower luminosity (see also Kumar \& Barniol Duran 2009a). They are then more difficult to detect. The best candidates for a LAT detection are therefore bursts with large $\Gamma_{0}$, because this ensures an early onset of the afterglow, implying large luminosities.

\section{Test of the Lorentz-invariance violation}

The arrival time of the $30 \mathrm{GeV}$ photon coincides with the peak of the afterglow emission. This is reasonable, because this is the time when we have the maximum probability to detect it (maximum flux and hard spectrum). If we assume that the $30 \mathrm{GeV}$ photon was indeed produced at the peak time, then the maximum possible time delay it can have is of the order of the width of the time bin of the peak $(0.15 \mathrm{~s})$. More conservatively, we can assume that the $30 \mathrm{GeV}$ photon was produced right at the beginning of the afterglow, and it arrives delayed by $0.22 \mathrm{~s}$ due to violation of the Lorentz invariance.

The time delay $\Delta t$ between the arrival time of a low and a high energy photon for a linear dependence of the photon's propagation speed on its energy is

$\Delta t=\frac{\Delta E}{M_{\mathrm{QG}} c^{2}} \int_{0}^{z} \frac{1+z}{H_{0} \sqrt{\Omega_{m}(1+z)^{3}+\Omega_{\Lambda}}} \mathrm{d} z$,

where $\Delta E$ is the difference between the low and high photon energy and $M_{\mathrm{QG}}$ is the quantum-gravity mass (A09; Amelino-Camelia et al. 1998; Jacob \& Piran 2008).

For a delay of $0.15 \mathrm{~s}$ we derive $M_{\mathrm{QG}}>6.7 M_{\text {Planck }}$, while the more conservative limit (delay of $0.217 \mathrm{~s}$ ) is $M_{\mathrm{QG}}>4.7 M_{\text {Planck }}$ (we used $h_{0}=0.71, \Omega_{\Lambda}=0.73, \Omega_{\mathrm{M}}=0.27$ ). These limits are consistent with those of A09, but exclude their lowest estimates.

\section{Conclusions}

The detection of an early high energy emission in the $\mathrm{GeV}$ range inevitably implies a large bulk Lorentz factor $\Gamma_{0}$ : to avoid suppression of the $\mathrm{GeV}$ emission due to the $\gamma-\gamma \rightarrow e^{ \pm}$process if the high energy photons belong to the prompt phase, or to have an early peak flux time if the emission belongs to the afterglow phase.

We have shown that the latter case is indeed favored (see also Gao et al. 2009), because we see the peak time of the emission in the LAT light-curve (as also seen in other GRBs in the infrared-optical band, e.g. Molinari et al. 2007 for GRB 060418 and GRB 060607A). Furthermore, also the energy spectral index $F(v) \propto v^{-1}$ is very similar to what we see in the afterglow phase. A large $\Gamma_{0}$, implying an early onset of the afterglow, means a large luminosity at the peak time (for equal emitted energy), and high typical frequencies. This makes synchrotron the most likely process for the LAT emission we see.

GRBs with smaller $\Gamma_{0}$ will have their prompt emission less blue-shifted, and it would be more difficult for them to reach the LAT energy range during their prompt phase. Their afterglows can achieve this, through the SSC process, but their afterglow peak time is longer, and so their fluxes are fainter (as $t_{\text {peak }}^{-1} \propto \Gamma_{0}^{8 / 3}$ if they emit the same amount of energy at the peak, see Eq. (2)). A large $\Gamma_{0}$, instead, means a large blue-shift for the photons of the prompt, an early onset of the afterglow, implying more flux at the peak, and finally higher intrinsic afterglow frequencies, allowing even the synchrotron photons of the afterglow to reach the LAT energy range. Therefore GRBs with large $\Gamma_{0}$ should be much more luminous in the LAT energy range than the other GRBs (as suggested also by Kumar \& Barniol Duran 2009a).

The limits derived here on the quantum gravity mass scale are not very different from those derived by A09, but we could associate the GBM and the LAT fluxes to two different components. We can then argue that the high energy photons are generated at (slightly) later times than the photons detected by the GBM, and the delay of their arrival times is not entirely due to quantum gravity effects. Instead, since photons above $100 \mathrm{MeV}$ belong to the same component, they are the best tool to investigate quantum gravity effects.

This suggests a recipe for a robust test on the Lorentz invariance violation, possible with very bright and short bursts detected at high energies. A short duration of the prompt ensures that the fireball has a relatively narrow width, and in turn this should correspond to a well-defined afterglow peak. A bright flux ensures good photon statistics, enabling us to measure more accurately possible delays as a function of photon energies.

Acknowledgements. We thank the referee P. Kumar for his useful comments. This research was supported by PRIN-INAF 2007 and ASI I/088/06/ grants. G. Ghirlanda acknowledges the NORDITA program on Physics of relativistic flows. We thank F. Tavecchio and Y. Poutanen for helpful discussions.

\section{References}

Abdo, A. A., Ackermann, M., Ajello, M., et al. 2009a, Nature, 462, 331 Abdo, A. A., Ackermann, M., Arimoto, M., et al. 2009b, Science, 323, 1688 Amelino-Camelia, G., Ellis, J., Mavromatos, N. E., Nanopoulos, D. V., \& Sarkar, S. 1998, Nature, 393, 763

Asano, K., Giurec, S., \& Meszaros, P. 2009, ApJ, 705, L191

Corsi, A., Guetta, D., \& Piro, L. 2009a, A\&A, submitted [arXiv:0905.1513] Corsi, A., Guetta, D., \& Piro, L. 2009b, ApJ, submitted [arXiv: 0911.4453]

Dado, S., \& Dar, A. 2009, ApJ, submitted [arXiv: 0910.0687] De Pasquale, M., et al. 2009, ApJ, 709, L146

Fan, Y.-Z., Piran, T., Narayan, R., \& Da-Ming, W. 2008, MNRAS, 384

Fishman, G. J., \& Meegan, C. A. 1995, ARA\&A, 33, 415

Gao, W.-H., Mao, J., Xu, D., \& Fan, Y.-Z. 2009, ApJ, 706, L146

Ghisellini, G., Ghirlanda, G., Nava, L., \& Celotti, A. 2009, MNRAS, in press [arXiv: 0910.2459]

Giuliani, A., Fuschino, F., Vianello, G., et al. 2009, ApJ, 708, L84 Golenetskii, S., Aptekar, R., Mazets, E., et al. 2009, GCN, 9344

Guiriec, S., Connaughton, V., Briggs, M., et al. 2009, GCN, 9336 Hoversten, E. A., Barthelmy, S. D., Burrows, D. N., et al. 2009, GCN, 9331

Jacob, U., \& Piran, T. 2008, PhRvD, 78, Issue 12

Kumar, P., \& Barniol Duran, R. B. 2009a, MNRAS, 400, L75

Kumar, P., \& Barniol Duran, R. B. 2009b, MNRAS, submitted [arXiv: 0910.5726]

Longo, F., Moretti, E., Barbiellini, G., et al. 2009, GCN, 9343

Ohmori, N., Noda, K., Sonoda, E., et al. 2009, GCN, 9355

Molinari, E., Vergani, S. D., Malesani, D., et al. 2007, A\&A, 469, L13

Piran, T. 2005, RvMP, 76, 1143

Rau, A., McBreen, S., Kruehlerv, T., et al. 2009, GCN, 9353

Razzaque, S., Dermer, C. D., \& Finke, J. D. 2009, ApJ, submitted [arXiv:0908.0513]

Sari, R., \& Piran, T. 1999, ApJ, 520, 641

Zhang, B., \& Peer, A. 2009, ApJ, 700, L65

Zou, Y.-C., Fan, Y.-Z., \& Piran, T. 2009, MNRAS, 396, 1163 